\title{
Neutron single target spin asymmetries in SIDIS (at JLab Hall A)
}

\author{
Evaristo Cisbani ${ }^{* \dagger}$ \\ INFN Rome - Sanità Group and Italian National Institute of Health \\ E-mail: evaristo.cisbani@iss.infn.it
}

The experiment E06-010 in Hall A at Jefferson Lab took data between November 2008 and February 2009 to directly measure, for the first time, the pion (and kaon) single "neutron" target-spin asymmetry (SSA) in semi-inclusive DIS from a polarized ${ }^{3} \mathrm{He}$ target.

Collins, Sivers (and Pretzelosity) neutron asymmetries are going to be extracted from the measured SSA.

Details of the experiment are described together with the preliminary results of the ongoing analysis.

Near future Hall A experiments on transverse nucleon spin structure are shorty reviewed.

XVIII International Workshop on Deep-Inelastic Scattering and Related Subjects April 19 -23, 2010

Convitto della Calza, Firenze, Italy

\footnotetext{
*Speaker.

†n behalf of the Hall A $6 \mathrm{GeV}$ Transversity Collaboration
} 


\section{Introduction}

The primary goal of the semi-inclusive deep inelastic electron scattering (SIDIS) E06-010 "Transversity" experiment at JLab Hall A [1]:

$$
e^{\rightarrow}+{ }^{3} \mathrm{He}^{\uparrow} \rightarrow e^{\prime}+h+X
$$

(with longitudinally polarized beam and transversely polarized target, $h=\pi$ or $K$, and $X$ undetected) is the extraction of the transverse single spin asymmetries $\left(A_{U T}\right)$ of positive and negative pion and kaon on neutron in the deep inelastic region. $A_{U T}$ is defined by:

$$
A_{U T} \equiv \frac{1}{\left|S_{T}\right|} \frac{d \sigma\left(\phi, \phi_{S}\right)-d \sigma\left(\phi, \phi_{S}+\pi\right)}{d \sigma\left(\phi, \phi_{S}\right)+d \sigma\left(\phi, \phi_{S}+\pi\right)} \equiv \frac{1}{\left|S_{T}\right|} \frac{d \sigma_{U T}}{d \sigma_{U U}}\left(\phi, \phi_{S}\right)
$$

being $S_{T}$ the transverse polarization of the target, $\phi$ and $\phi_{S}$ the azimuthal angles of the scattered hadron $h$ and the target spin relative to the lepton scattering plane.

In the current theoretical framework [2], at leading approximation in $O(1 / Q)$ (and dropping the $\cos 2 \phi$ term in $\left.d \sigma_{U U}\right), A_{U T}$ is reproduced by the linear combination of three asymmetries:

$$
\frac{d \sigma_{U T}}{\left|S_{T}\right|} \sim \underbrace{\left[\delta q \otimes H_{1}^{\perp}\right] \sin \left(\phi+\phi_{S}\right)}_{\text {Collins Asym. }}+\underbrace{\left[f_{1 T}^{\perp} \otimes D_{1}\right] \sin \left(\phi-\phi_{S}\right)}_{\text {Sivers Asym. }}+\underbrace{\left[h_{1 T}^{\perp} \otimes H_{1}^{\perp}\right] \sin \left(3 \phi-\phi_{S}\right)}_{\text {Pretzelosity Asym. }}+O(1 / Q)
$$

where the terms in the square bracket represent sums (on partons) of convolution integrals between Transverse Momentum Dependent (TMD) Distribution Function (DF) and Fragmentation Function (FF); the latter gives information on the hadron formation (and therefore on the peculiar QCD confinement); the former describes the dynamics of the partons inside the nucleon.

In SIDIS the DF and FF cannot be directly disentangled [3], however, with proper fit of the $\sin (. .$.$) modulation functions, the three asymmetries of 1.1$ can be extracted, as already done for the Collins and Sivers asymmetries in HERMES (on polarized proton) and COMPASS (on proton and deuteron), at different 4-momentum transfer $Q^{2}$ (see [4]) ${ }^{1}$. The results are not fully understood and partially inconsistent between the two experiments. In any case, a direct measure on the neutron, as proposed by the Transversity experiment is expect to be of valuable importance to shed light on the subject.

The data taken in the Transversity experiment also permits the measurement of the double spin asymmetry $A_{L T} \cos \left(\phi_{h}-\phi_{S}\right)$ proportional to the TMD $g_{1 T}^{\perp}$ convoluted with the unpolarized FF.

\section{Experimental Setup}

The Transversity experiment used the CEBAF longitudinally polarized (up to $80 \%$ with fast flipping of $30 \mathrm{~Hz}$ ) electron beam with $15 \mu \mathrm{A}$ current (limited by the target stability) at the maximum available $5.9 \mathrm{GeV}$ energy.

The beam diffused on the high pressure transversely polarized ${ }^{3} \mathrm{He}$ target which acts as effective neutron target, with a density of $50 \mathrm{mg} / \mathrm{cm}^{2}$ and $40 \mathrm{~cm}$ long, providing a luminosity of

\footnotetext{
${ }^{1}$ The Pretzelosity asymmetry has been preliminary measured by COMPASS, at low $x$ on deuteron for hadron and it seems consistent with zero [5].
} 
Table 1: Spectrometer settings and kinematics used in the Trasversity Experiment

\begin{tabular}{rcc}
\hline Particle & $\begin{array}{c}\text { BigBite } \\
\text { electron }\end{array}$ & $\begin{array}{c}\text { HRS-Left } \\
\text { hadron }\end{array}$ \\
Momentum & $P_{e^{\prime}}=0.7 \div 2.2 \mathrm{GeV} / \mathrm{c}$ & $P_{h}=2.35 \mathrm{GeV} / \mathrm{c} \pm 5 \%$ \\
Angle & $\theta_{e^{\prime}}=30^{\circ}, \Delta \Omega_{e^{\prime}}=64 \mathrm{mrs}$ & $\theta_{h}=16^{\circ}, \Delta \Omega_{h}=6 \mathrm{msr}$ \\
Kinematic Region & $\left\langle Q^{2}\right\rangle \sim 2.2 \mathrm{GeV}^{2}, x \sim 0.13 \div 0.45, z \sim 0.5$ \\
\hline
\end{tabular}

$10^{36}\left(\mathrm{~s} \cdot \mathrm{cm}^{2}\right)^{-1}$. The target operated at a record steady polarization of $65 \%$ obtained by spin exchange of K-Rb optically pumped by 3 narrow, high power laser lines. The direction of the polarization is controlled by three pairs of Helmholtz coils and flipped every 20 minutes. Polarization was measured during the experiment by Nuclear Magnetic Resonance based polarimetry, calibrated via Electron Paramagnetic Resonance [6].

The scattered electrons and hadrons were detected in coincidence by the BigBite and HRSLeft spectrometers respectively; relevant spectrometer settings and kinematics are summarized in table 1.

BigBite were equipped with a large dipole magnet, 3 drift chambers for a total of 18 planes for precise tracking (spatial resolution $\sigma=180 \mu \mathrm{m}$ ) and momentum reconstruction (in combination to the magnetic field). Pre-shower and shower were used for electron identification and pion rejection. Fast segmented scintillators were used for timing [6].

The HRS-Left, part of the Hall A standard equipment [7], with its complex optics for high resolution of the momentum of the particle, housed the tracking drift chambers, scintillator planes, and a sophisticated identification system consisting of:

- an aerogel Cherenkov counter $(n=1.015)$ and a RICH proximity focusing detector with liquid Freon radiator $(n=1.27)$ for hadron identification

- a gas Cherenkov radiator and a wall of Lead-glass blocks for electron-hadron identification.

\section{Hadron production}

Analysis of the data is carried on by two independent teams exploiting different extraction methods: local pair-angular bin-fit method ideal for pion asymmetry and maximum likelihood method [8]; first results on ${ }^{3} \mathrm{He}$ asymmetries are expected in the coming weeks.

One of the peculiarity of the experiment is the ability to identify the hadrons with high purity due to the exploitation of the Time of Flight, the Aerogel Cherenkov counter and the RICH detector. Preliminary results on the different hadron production and the ability to identify the $K$ and $p$ are presented in table 2 .

It is worth noting the lower production of kaons respect to the prediction of $10 \div 20 \%$ (of pions) based on the HERMES data; the worse purity of the negative kaons is caused by the higher relative population of pions. 
Table 2: Preliminary results of the hadron production and identification purity for both transverse polarization directions of the target.

\begin{tabular}{c|cc|c}
\hline & \multicolumn{2}{|c|}{ Target Transverse Polarization } & \\
& Vertical & In plane & Purity \\
\hline$\pi^{+}$ & $117 \mathrm{k}$ & $115 \mathrm{k}$ & \\
$K^{+}$ & $5.8 \mathrm{k}$ & $5.8 \mathrm{k}$ & $97.1 \%$ \\
$p$ & $126 \mathrm{k}$ & $126 \mathrm{k}$ & $97.2 \%$ \\
\hline$\pi^{-}$ & $82 \mathrm{k}$ & $91 \mathrm{k}$ & \\
$K^{-}$ & $0.99 \mathrm{k}$ & $1 \mathrm{k}$ & $94.2 \%$ \\
$\bar{p}$ & 61 & 68 & \\
\hline
\end{tabular}

\section{Future experiments}

The Transversity experiment has exploited the current resources at JLab at its best. However in the coming few years, the CEBAF beam energy will be doubled and the existing experimental Halls will be served by electron beam with high current (up to about $100 \mu \mathrm{A}$ ), high longitudinal polarization (up to $85 \%$ ) and maximum energy of $11 \mathrm{GeV}$. In this prospective, several new experiments have been proposed for precise and extensive measurements of the SSA both on proton and neutron. A quite extended TMD program is proposed in Hall B [9] while two new experiments are planned in Hall A, designed to improve both statistics and phase space coverage respect to the above described Transversity:

- SBS-SIDIS on $\pi$ and K (conditionally approved, 01/2009) [10]:

1. similar layout of the Transversity experiment but with higher acceptance and luminosity thanks to the replacement of the HRS-Left by SBS [11] a new large acceptance and very forward angle spectrometer and a quite improved ${ }^{3} \mathrm{He}$ target capable of supporting higher beam current;

2. will permit 2D binning of the SSA's on the relevant variables: $x, P_{\perp}$ and $z$, for both hadrons and different $Q^{2}$ values;

3. high $x$ valence region (with overlap to the existing data).

- SoLID-Transversity experiment (approved, 01/2010) [12]:

1. use SoLID a new spectrometer with a solenoid magnet configuration that will offer $2 \pi$ angular acceptance, providing an optimal control of systematics;

2. precision measurement in $4 \mathrm{D}$ phase space $\left(x, z, P_{\perp}\right.$ and $\left.Q^{2}\right)$

3. extended phase space coverage

The two experiments present complementary features, including expected time scales of data taking, due to the higher complexity of the SoLID equipment which will permits ultimate precision on the SSA extraction. 


\section{References}

[1] E06-010 proposals and home page: http://hallaweb.jlab.org/experiment/transversity/

[2] A. Bacchetta et al., JHEP 02 (2007) 093

[3] M. Burkardt, C.A. Miller, W-D Nowak, Reports on Progress in Physics 73 (2010) 016201

[4] H. Fisher, this proceedings; A. Martin, this proceedings; HERMES Collaboration, PRL 103 (2009) 152002; L. Pappalardo at Transversity 2008, Ferrara, Italy

[5] A. Kotzinian on beahalf of the COMPASS collaboration, arXiv:hep-ex.0705.2402

[6] Xin Qian, "Measurement of Single Spin Asymmetry in $n^{\uparrow}\left(e, e \pi^{ \pm}\right) X$ on Transversely Polarized ${ }^{3} \mathrm{He} "$, PhD Thesis, Duke University, 2010

[7] J. Alcorn et al., NIM A522 (2004) 294-346

[8] J. Huang, Technical note available at http://www.jlab.org/ jinhuang/Transversity/MLE.pdf

[9] P. Rossi, this proceedings

[10] G. Cates, E. Cisbani, G.B. Franklin and B. Wojtsekhowski spokespeople, PR09-018 PAC34 Conditionally Approved proposal, http://hallaweb.jlab.org/collab/PAC/PAC34/PR-09-018-sidis.pdf

[11] SBS web page http://hallaweb.jlab.org/12GeV/SuperBigBite/

[12] J.-P. Chen, H. Gao, X. Jiang, J.-C. Peng spokespeople, E10-006 PAC35 Approved experiment, http://hallaweb.jlab.org/collab/PAC/PAC35/PR-10-006-SoLID-Transversity.pdf 